\title{
El problema de marco y dos programas rivales en psicología cognitiva
}

\section{The Frame Problem and Two Rival Programs in Cognitive Psychology}

\author{
RODRIGO MORO Y MARÍA INÉS SILENZI \\ Universidad Nacional del Sur (Argentina)
}

Recibido: 23/01/2016 Aceptado:08/04/2016

\begin{abstract}
RESUMEN
El problema de marco es un problema central de las Ciencias Cognitivas. En el ámbito filosófico, se ha discutido si el uso de heurísticas permitiría solucionar dicho problema. Aquí nos enfocamos en las investigaciones psicológicas sobre el tema. El problema es que no hay un único programa de investigación sobre heurísticas sino dos: el programa de Heurísticas y Sesgos y el programa de Heurísticas Rápidas y Frugales. Analizamos, así, las capacidades relativas de ambos programas para promover una solución al problema de marco. Argumentamos que el programa de Heurísticas Rápidas y Frugales parece ser más adecuado para ese propósito.

PALABRAS CLAVE

PROBLEMA DE MARCO, HEURÍSTICAS, PROGRAMA DE HEURÍSTICAS Y SESGOS. PROGRAMA DE HEURÍSTICAS RÁPIDAS Y FRUGALES.

ABSTRACT

The frame problem is a central problem in Cognitive Sciences. In philosophy, researchers have discussed whether the use of heuristics would solve such a problem. Here we focus on the psychological research on this issue. The problem is that there is not a unique research program on heuristics but two: the Heuristics and Biases Program and the Fast and Frugal Heuristics Program. We analyze, thus, the relative abilities of both programs to provide a solution to the frame problem. We argue that the Fast and Frugal Heuristic Program seems to be more adequate for this purpose.
\end{abstract}

(C) Contrastes. Revista Internacional de Filosofía, vol. XXII-Nº 1 (2017), pp. 101-117. ISSN: 1136-4076 Departamento de Filosofía, Universidad de Málaga, Facultad de Filosofía y Letras Campus de Teatinos, E-29071 Málaga (España) 


\section{KEYWORDS \\ FRAME PROBLEM, HEURISTICS, HEURISTICS AND BIASES PROGRAM, FAST AND FRUGAL HEURISTICS PROGRAM}

\section{INTRODUCCIÓN}

El PROBLEMA DE MARCo (McCarthy y Hayes 1969) surgió dentro del campo de la Inteligencia Artificial como el problema acerca de cómo representar, de la forma más concisa posible, el hecho de que, en una situación determinada, la mayoría de las propiedades de los objetos permanecen iguales o no son afectadas por la ejecución de una determinada acción (Shanahan 1997). A pesar de su origen lógico, no pasó mucho tiempo para que el problema de marco sea abordado por varios filósofos (Dennett 1984, Fodor 1986, Brown 1987, Pylyshyn 1987, Ford y Hayes 1991, Ford y Pylyshyn 1996, entre otros) siendo motivo en las décadas de los $80^{\prime}-90^{\prime}$ de grandes debates y controversias dentro del campo de las Ciencias Cognitivas.

Sin embargo, este problema es aún hoy es un punto de partida para la discusión de varios desacuerdos entre investigadores de distintas áreas. Uno de los motivos de tales enfrentamientos gira en torno a las múltiples interpretaciones del problema de marco que proponen lógicos, filósofos y recientemente, investigadores cognitivos. En efecto, al examinar la literatura sobre el problema de marco, se puede observar que la mayoría de las veces se menciona, especialmente al principio de cada tratamiento particular del problema, la complicación de definirlo (Silenzi 2015). Pese a esta dificultad, Silenzi (2014) muestra que se puede encontrar cierta convergencia entre las distintas definiciones del problema, interpretándolo finalmente como un conjunto de problemas relacionados con la determinación de relevancia ${ }^{1}$ (Glymour 1987, Crockett 1994, Dreyfus 1979, Schneider 2007, Pinker 2005, Shanahan 2009; Ludwing y Schneider 2008, entre otros).

De acuerdo a esta interpretación, y en el contexto de la realización de una tarea, el problema de marco cuestiona cómo un sistema cognitivo, que posee una enorme cantidad de información, selecciona la información relevante

1 Parece necesario establecer, antes de continuar, el significado del concepto de «relevancia» que utilizaremos a lo largo del trabajo, ya que dicho término suele considerarse, con justicia, polisémico. Dado que el problema de marco siempre se plantea en el contexto de la realización de una tarea y toda tarea implica algún objetivo a satisfacer, consideramos que la definición presentada por Hjørland (2010) es especialmente adecuada: «algo (x) es relevante para una tarea (T), si x aumenta la probabilidad de lograr el objetivo (O) que es implicado por T» (Hjørland 2010, p. 229, traducción propia). 
para la realización de la tarea en cuestión en un tiempo acotado y con recursos computacionales limitados. En particular, uno de los aspectos particulares del problema de marco se centra en la pregunta de cómo, de hecho, los seres humanos realizan esta selección de información relevante. Nuevamente, esta pregunta no agota el problema de marco sino que es uno de los aspectos que integran el conjunto de preguntas abiertas en el problema de marco. Silenzi \& Moro (2015) argumentan que, para responder esta pregunta, serán claves los aportes de la psicología cognitiva, ya que los investigadores de dicha disciplina tienen por objetivo lograr buenos modelos explicativos acerca de cómo funciona la mente humana.

Teniendo en cuenta estas consideraciones es menester resaltar que la literatura del área da por supuesto que las personas suelen determinar qué es relevante para una tarea dada de manera rápida y adecuada, aunque, por supuesto, de vez en cuando surjan errores. Llamaremos a esta regularidad el «fenómeno fundamental» de la literatura sobre el problema de marco. No parece plausible atribuirle al azar la prontitud y el nivel de éxito logrado y, por lo tanto, una pretendida solución de este aspecto del problema de marco debería dar cuenta del mencionado fenómeno fundamental; más específicamente, debería dar cuenta de cómo a las personas parece «venirse a la mente» la información relevante para una tarea de entre una cantidad desmesurada de información que poseen, y eso, además, en el momento preciso y de manera adecuada.

Es a propósito de este particular aspecto del problema de marco (llamémoslo «aspecto psicológico») que entra en consideración nuestra cuestión clave: la aptitud del uso de heurísticas para resolverlo. Las heurísticas son reglas prácticas que, usando poca información, proveen soluciones razonables a problemas de razonamiento y decisión. Así, se ha discutido en el ámbito filosófico si el uso de heurísticas sería la herramienta cognitiva fundamental para aliviar la carga computacional en la determinación de la relevancia y con ello, para resolver el problema de marco (Carruthers 2006a, 2006b, Fodor 2008, Samuels, 2004, 2005, 2010). El objetivo de este trabajo es explorar las investigaciones en psicología cognitiva sobre el tema. El problema que encontramos es que no hay un único programa de investigación sobre heurísticas. Más precisamente, hay dos programas de investigación rivales, el programa de Heurísticas y Sesgos (liderado por Daniel Kahneman) y el programa de Heurísticas Rápidas y Frugales (liderado por Gerd Gigerenzer) que apelan al uso de heurísticas para explicar regularidades empíricas de razonamiento y decisión (Gilovich et al. 2002, Gigerenzer y Selten 2001). Así, el objetivo principal de este trabajo es analizar las capacidades relativas de ambos programas para promover una solución al problema de marco. Después de presentar cada programa (sección II), revisaremos qué dicen explícitamente 
los psicólogos cognitivos de ambos programas acerca del problema de marco (sección III). Posteriormente, argumentaremos que si bien, en principio, ambos programas pueden proveer una contribución a dicho objetivo, el programa de Heurísticas Rápidas y Frugales parece ser más adecuado para ese propósito (sección IV). Finalmente, presentaremos nuestras conclusiones (sección V).

\section{DoS PROGRAMAS RIVALES SOBRE HEURÍSTICAS}

Ciertamente, el lector podría desafiar nuestra afirmación de que el programa de Heurísticas y Sesgos y el programa de Heurísticas Rápidas y Frugales son genuinamente programas rivales. En particular, pueden ser concebidos como variantes de un solo programa difiriendo en los problemas analizados. Sin embargo, nuestra afirmación de que se trata de programas rivales se basa en las propias declaraciones de los protagonistas (Gigerenzer 1996; Kahneman y Tversky 1996). Adicionalmente, veremos posteriormente que diferencias en metodología y forma de trabajo de dichos programas van a tener consecuencias cruciales a la hora de compararlos con respecto a su potencial contribución al problema de marco. A continuación, presentaremos brevemente cada uno de los programas.

\section{II.1. El PROGRAMa DE HeURísticas y SESGoS}

Este programa fue iniciado por Tversky y Kahneman en los años 70 (véase un compendio en Gilovich, Griffin y Kahneman 2002). Actualmente, el programa defiende el enfoque de procesamiento dual de información. Nuestras mentes serían procesadores de información, pero tendrían dos sistemas funcionando simultáneamente, en lugar de uno. El sistema 1 sería automático, requiriendo poco esfuerzo cognitivo, de carácter asociativo, de funcionamiento paralelo, de procesamiento opaco. Por otra parte, habría un sistema 2 con las características opuestas: controlado, requiriendo mucho esfuerzo cognitivo, deductivo, serial, auto-consciente. Las heurísticas serían estrategias del sistema 1.

La idea es que las personas utilizan heurísticas para elaborar juicios y tomar decisiones que involucran situaciones de incertidumbre. La ventaja es que, comparado con modelos normativos (a continuación aclararemos este concepto), las heurísticas reducen el tiempo y el esfuerzo para realizar juicios y decisiones. La desventaja es que, en determinadas circunstancias o contextos, estas heurísticas conducen a errores sistemáticos o sesgos. Para determinar si la respuesta de un participante es aceptable o errónea, este programa suele usar como estándares normativos la lógica, el cálculo de probabilidades y la teoría de la decisión. Stein (1996) llama a esta postura «concepción estándar de racionalidad». Utilizando implícitamente esta concepción y ante los resultados experimentales que muestran una gran 
variedad de sesgos, algunos investigadores sacaron conclusiones pesimistas acerca de la racionalidad humana (véase, por ejemplo, Kanwisher 1989 y Piattelli-Palmarini 1991). Sin embargo, Tversky y Kahneman siempre se mantuvieron neutrales ante tal discusión (véase, especialmente, el prólogo de Gilovich et al., 2002). Más concretamente, proponen centrarse en los errores por una cuestión metodológica: al igual que en las ilusiones ópticas, en los sesgos se aprecia mejor el funcionamiento de los procesos de elaboración de juicios y decisiones. Para lograr apreciar las investigaciones de este programa, es necesario entrar en los detalles. Comentaremos, a continuación, una de las heurísticas más trabajadas por el programa de Heurísticas y Sesgos: la heurística de representatividad (representativeness).

En general, las heurísticas se activan al presentar a los participantes determinadas tareas. En el caso de la heurística de representatividad se postula que se activa ante la tarea de determinar la probabilidad de que determinado objeto pertenezca a cierta categoría, por ejemplo que una persona con ciertas características sea de determinada nacionalidad/profesión/inclinación política. Básicamente, para juzgar la probabilidad de que A sea un B, la heurística utiliza el grado en que A sea representativo de B o el grado en que A se asemeje a B. Más específicamente, según Kahneman y Frederik (2002), la heurística consiste en realizar dos sustituciones: 1) la categoría se reemplaza por un prototipo o ejemplar de dicha categoría; y 2) la evaluación de probabilidad se reemplaza por evaluación de similitud del objeto con el prototipo. Por ejemplo, los participantes reciben la siguiente información.

Un panel de psicólogos ha entrevistado a 30 ingenieros y 70 abogados. Basándose en esa información, se han elaborado descripciones cortas de esas 100 personas. Para las siguientes descripciones, indique con qué probabilidad cree que la persona es un ingeniero, usando una escala de 0 a 100.

Jack tiene 45 años, es casado con 3 hijos. Es generalmente muy detallista y ambicioso. No le interesan los temas políticos ni sociales y emplea la gran mayoría de su tiempo libre con sus hobbies que incluyen carpintería y la resolución de problemas matemáticos.

A continuación, se les pregunta a los participantes sobre la probabilidad de que tal persona sea ingeniero. Las personas no tienen problemas para responder prontamente este tipo de preguntas y la evidencia apunta a que utilizan la heurística de representatividad para hacerlo. En el ejemplo dado los participantes suelen considerar que el perfil dado es similar al prototipo de ingeniero (respaldado por grupos de participantes independientes) y por eso suelen hacer estimaciones muy altas de la probabilidad de que dicho sujeto sea ingeniero (Tversky y Kahneman 1974). Nótese que tal inferencia es razonable y se realiza de manera extremadamente rápida. Adicionalmente, 
es claro que la información utilizada es relevante para responder la pregunta. Así, vemos cómo el uso de esta heurística contribuye a solucionar el problema de marco: la tarea dispara el uso de una heurística que requiere la búsqueda de información muy precisa (prototipos de las distintas profesiones) y el procesamiento de la información es también muy sencillo (similitud entre el perfil y los distintos prototipos). Sin embargo, el programa de Heurísticas y Sesgos postula que el uso de tal heurística permite también dar cuenta de varios sesgos de razonamiento y decisión (en las secciones 3 y 4 veremos esto en detalle y analizaremos sus consecuencias). No obstante, lo presentado permite mostrar que, en principio, es plausible que el enfoque de Heurísticas y Sesgos pueda contribuir a la investigación sobre el aspecto psicológico del problema de marco.

Antes de presentar el enfoque rival al programa de Heurísticas y Sesgos, es menester señalar la existencia de un nuevo paradigma de investigación en el área de psicología del razonamiento. Este nuevo paradigma, liderado por Jonathan Evans y David Over, enfatiza el rol del juicios de probabilidad y de utilidad en el razonamiento humano y busca integrar la investigación de psicología del razonamiento con la psicología de la elaboración de juicios y toma de decisiones (véase una presentación detallada en Elqayan y Over, 2013). De interés para nuestros propósitos, este nuevo paradigma también postula que las personas utilizan heurísticas al realizar tareas. Sin embargo, aunque novedoso en muchos aspectos, este nuevo paradigma comparte exactamente la misma concepción de heurísticas del programa de Heurísticas y Sesgos. En particular, postulan que el uso de heurísticas genera, en ciertos contextos, errores sistemáticos de juicio y decisión (véase, por ejemplo, los estudios de Blanchete y Caparos, 2013 y Thompson et al., 2013). Así, para evitar redundancias, dejaremos de lado el análisis detallado de los desarrollos de este nuevo paradigma.

\section{II.2. EL PROGRAMa DE HEURÍSTICAS RÁPIDAS Y FRUGALES}

Gerd Gigerenzer lidera un programa rival de investigación que realiza muchas críticas metodológicas al programa de Heurísticas y Sesgos (véase, por ejemplo, Gigerenzer 1996). Si bien Gigerenzer y sus colaboradores concuerdan con el hecho de que las personas utilizan heurísticas para razonamientos y decisiones, se manifiestan en desacuerdo con que el uso de las mismas genere sesgos. En general, este programa ve los sesgos como artefactos de los procedimientos experimentales del programa rival y, en consecuencia, tiene una postura relativamente optimista con respecto a la racionalidad humana. Gigerenzer y Selten (2001) justamente defienden una noción de racionalidad que toma en cuenta el factor adaptación al ambiente. Ellos llaman a esta concepción «racionalidad ecológica». Gigerenzer señala 
que esta noción de racionalidad debería ser clasificada como de racionalidad acotada, ya que toma en consideración que los agentes humanos tienen recursos limitados en tiempo, conocimiento y poder computacional. ${ }^{2}$ Además de los factores de tiempo escaso y limitaciones cognitivas, esta concepción sostiene que los ambientes tienen cierta estructura informacional, es decir, que proveen información acerca de algunos aspectos (aunque, crucialmente, no de todos) y que esta información es dada en un formato específico. Al concentrarse en estos puntos, este enfoque estudia el acople entre heurísticas usadas por humanos y estructuras ambientales.

Los criterios de racionalidad usados son efectividad, frugalidad y velocidad. ${ }^{3}$ Efectividad se refiere a la relación entre juicios o predicciones y realidad; frugalidad se refiere a la cantidad de información que una heurística dada necesita para operar. Finalmente, velocidad se refiere a la manera en la cual la heurística dada procesa la información (cuanto más simple este proceso, más rápido el juicio o decisión).

Como resultado de sus investigaciones, el nuevo programa postula un conjunto distinto de heurísticas. Por razones de espacio, sólo presentaremos una de las más trabajadas por el programa, a saber, la heurística denominada «toma lo mejor» (take the best).

Se postula que esta heurística se activa cuando se tiene que decidir cuál de dos objetos es superior al otro con respecto a cierto criterio y hay pistas sobre diferentes aspectos que sugieren un resultado u otro. Lo que la heurística hace es determinar cuál de las pistas tiene mayor validez (brinda mayor proporción de respuestas correctas) y utilizar solamente dicha pista para tomar la decisión en cuestión. Si dicha pista no genera una respuesta (i.e., hay empate entre los objetos) se considera la segunda pista con mayor validez y así sucesivamente (Gigerenzer et al. 2002). Por ejemplo, considérese la tarea de determinar cuál de dos ciudades tiene una mayor tasa de personas-sin-techo (homeless). Para contribuir a la decisión, se les da previamente a los participantes información sobre otros pares de ciudades con detalles acerca de dicha tasa en adición a información sobre desempleo, pobreza, refugios públicos, clima, etc. en

2 La concepción de racionalidad estándar - defendida implícitamente por Tverky y Kahneman- es, por contraste, una concepción de racionalidad ilimitada porque sus principios son postulados sin ninguna consideración de posibles limitaciones humanas, esto es, estos principios son postulados que operan como si los agentes dispusieran de tiempo y poder computacional infinitos.

3 Una característica interesante de este enfoque es que no toma consistencia o propiedades similares como condiciones sine qua non para decisiones racionales. Esto implica que hay situaciones donde decisiones inconsistentes podrían ser perfectamente racionales. Gigerenzer y Selten (2001) expresan este punto de la siguiente manera: «cuando ser impredecible es adaptativo, preferencias consistentes pueden ser letales» (p. 41, traducción propia). 
cada una de las ciudades. Lo que tienden a hacer las personas, entonces, es utilizar la heurística toma lo mejor, es decir, determinan cuál de los aspectos tiene mayor poder predictivo y utilizan sólo ese aspecto para tomar la decisión (Newell et al. 2003, Gigerenzer 2008). Análisis formales muestran que en muchos contextos la heurística tiene una efectividad levemente inferior al modelo bayesiano ${ }^{4}$ pero utiliza mucha menos información, es decir, es más frugal y la procesa de manera más simple, es decir, es más rápida (Gigerenzer et al. 2002). E incluso hay contextos (por ej., cuando se limita severamente la información previa sobre otras ciudades), donde la heurística predice mejor que los modelos formales (Gigerenzer y Brighton 2009).

Analicemos, entonces, el funcionamiento de la heurística comentada en relación a la determinación de relevancia. Nótese que el ranking de pistas que ordena realizar la heurística puede tomarse como un ranking de relevancia: en efecto, se busca como criterio de decisión la pista que mayor poder predictivo tenga, lo cual puede ser visto como la pista más relevante al momento de hacer una predicción. Y lo mismo que se dijo con respecto a la heurística representatividad, vale acá también: básicamente, la heurística toma lo mejor también demanda una búsqueda restringida de información y la procesa de manera sencilla y eso evita que el agente se pierda en un mar de información, y pueda así resolver prontamente y de manera razonable la tarea. Por lo tanto, parece plausible que las investigaciones de este programa también contribuyan a proveer una solución al problema de marco.

Es importante notar, antes de finalizar esta sección, que este programa de investigación difiere del anterior en un punto importante. Además de ofrecer un conjunto distinto de heurísticas, la posición global es que en general las personas usan heurísticas adecuadas para resolver problemas, es decir, heurísticas que no tienden a generar errores sistemáticos. En la siguiente sección veremos que este punto será crucial para la comparación entre ambos programas.

\section{III. ¿QUÉ DICEN LOS PSICÓLOGOS COGNITIVOS DE LOS DIFERENTES PROGRAMAS SOBRE EL PROBLEMA DE MARCO?}

Teniendo en cuenta la existencia de estos dos programas, surge inmediatamente la siguiente pregunta: ¿ambos programas son igualmente adecuados para promover una solución al problema de marco? Ante esta pregunta, el primer recurso a explorar es averiguar qué dicen los representantes de ambos programas acerca del problema de marco. La respuesta resumida es la siguiente: prácticamente nada. El problema de marco no ha sido estudiado

4 El modelo bayesiano básicamente utiliza toda la información disponible y la integra aplicando el cálculo de probabilidades. 
en profundidad en psicología cognitiva, aunque ha recibido algunas menciones esporádicas.

Shaffir y Tversky (1992) lo mencionan para apoyar la tesis de que las personan no razonan de manera «extensional», en el sentido de analizar todas las posibilidades abiertas en una decisión con la posterior comparación de las consecuencias de cada una. Reconocen que, gracias a ello, el problema de marco no es un problema para los seres humanos y sí lo es para las computadoras, en el sentido de que con gran cantidad de información ese tipo de análisis demandaría recursos cognitivos y temporales extraordinarios.

De manera similar, Gigerenzer (2000) realiza sólo una breve mención del tema, especulando que si la selección natural hubiera generado un sistema cognitivo de reglas generales como las bayesianas, se habría topado con el problema de marco; es decir, aclara, con el problema de explosión combinatoria. Así, lo menciona para defender su posición de que la inteligencia social es modular, como opuesta a la de un procesador central que maneja reglas generales - a la Fodor.

Finalmente, Evans (2009) -cercano al programa de Heurísticas y Sesgosafirma que el uso de heurísticas soluciona el problema de marco, en el sentido de que contextualizan nuestro pensamiento, recuperando creencias y otros elementos de la memoria que son relevantes para el contexto en cuestión, aunque a veces puedan conducir a ciertos sesgos. Pese a pronunciarse sobre nuestro tema de interés, no desarrolla su posición en detalle.

El artículo de Ketellar y Todd (2001) -quienes pertenecen al programa de Gigerenzer- es, a nuestro conocimiento, el único artículo donde psicólogos cognitivos expertos en heurísticas se centran en el problema de marco. A continuación lo analizaremos en detalle, pero una conclusión parcial es clara: aunque los principales referentes de ambos programas tienen presente, al menos lateralmente, el problema de marco, es prácticamente nula la investigación específica sobre el tema. Así, nos parece importante recalcar que sería deseable una mayor profundización en la investigación sobre el tema, tanto en el análisis como en la recolección de evidencia empírica.

Para ofrecer una respuesta sobre la comparación de los programas, comenzaremos resumiendo la posición de Ketelaar y Todd (2001), como decíamos, único trabajo explícito sobre el tema. Como era de esperarse, establecen una posición contrapuesta a la de Tversky y Kahneman. En particular, se centran en la heurística denominada «pico-fin» (peak-end rule), postulada por Kahneman y colaboradores (véase un compendio en Kahneman 1999). La evidencia empírica apunta a que las personas utilizarían dicha heurística para ponderar experiencias cortas (un procedimiento médico, una película). Básicamente, lo que se muestra es que las personas tienden a juzgar la calidad de una experiencia (positiva o negativa) por el promedio entre su pico 
de placer o displacer y su final. Nuevamente, para entender la discusión, hay que entrar en los detalles. Esta heurística -según Kahneman y colaboradoresda lugar a sesgos de juicio y decisión, ya que, por ejemplo, hay un descuido del tiempo de duración de la experiencia (duration neglect). Se comenta, por ejemplo, el estudio de Redelmier y Kahneman (1996) realizado con pacientes sometidos a una colonoscopia o a una litotricia. Durante dicho procedimiento médico, y una vez por minuto, se les pedía a los participantes que reportaran el nivel de dolor que iban experimentando. Después de una hora finalizado el procedimiento, se les pedía a los pacientes una evaluación global del nivel de dolor. El modelo que mejor predecía los valores de la evaluación global era el de la heurística propuesta, es decir, el que promediaba el valor del pico de dolor con el valor del momento final. Sin embargo, la duración del procedimiento no tuvo ninguna influencia en las evaluaciones globales. Esto es extremadamente llamativo, ya que el rango de duración de los procedimientos iba desde 4 a 69 minutos. En otro estudio también se mostró que, en algunos contextos, las personas tienden a elegir opciones que los llevan a un grado absoluto mayor de displacer, ya que si a la misma experiencia negativa se le agrega un periodo posterior donde el displacer disminuye, esta última opción suele ser preferida a la primera. Esto es mostrado en el estudio de Kahneman et al. (1993), donde los participantes pasaban por dos experiencias: 1) sumergir su brazo en agua fría $\left(14^{\circ} \mathrm{C}\right)$ durante un minuto y 2$)$ lo mismo más 30 segundos donde el agua era levemente entibiada $\left(15^{\circ} \mathrm{C}\right)$-aunque algunos participantes ni siquiera notaban la diferencia. Cuando se les decía a los participantes que debían repetir uno de los procedimientos, alrededor del 70\% elegía el segundo.

Ahora bien, Ketelaar y Todd se oponen a Kahneman y colaboradores sobre si deben extraerse consecuencias negativas del uso de esta heurística. No niegan que las personas utilicen de hecho esa heurística sino que lo aceptan sin problemas. Lo que afirman es que, en muchas condiciones ecológicas, tiene sentido afirmar que no importa cuánto dura una experiencia sino cómo termina, por ejemplo, en una partida de ajedrez. Por eso, en condiciones de escasez de recursos temporales y cognitivos, enfocarse en el final no parece en absoluto irracional. En cuanto a enfocarse en el «pico o cúspide» de una experiencia, afirman que en muchas situaciones ecológicas tenemos que elegir entre estrategias que son placenteras en el corto plazo pero muy malas en el largo plazo y estrategias que requieren un pequeño sacrificio inicial pero que son muy positivas a largo plazo. Así, nuestra tendencia a elegir experiencias donde el pico de displacer esté al principio tampoco serían irracionales.

Aunque los argumentos de Ketelaar y Todd parecen ciertamente razonables, no son del todo convincentes. En primer lugar, poner en el mismo nivel los dos factores clave, desde el punto de vista normativo, es discutible. Es cierto que en casi todas las situaciones importa tremendamente cómo finaliza 
una experiencia, por lo que tener en cuenta el final parece indiscutiblemente sensato. Por otra parte, en cuanto al pico de placer y/o displacer, es cierto que parece razonable ser sensible a su ubicación y, así, a la tendencia general de la experiencia. Sin embargo, no es claramente obvio que tal factor deba ser tenido en cuenta en toda evaluación global de experiencia. En segundo lugar, y éste es el punto clave, la defensa es muy pobre; básicamente, diría que así como hay situaciones donde el uso de la heurística genera errores sistemáticos, habría situaciones -descriptas vagamente- donde el uso de la heurística no genera errores. Pero no se ofrece ningún apoyo experimental que muestre que el uso de la heurística en cuestión tienda a generar respuestas acertadas en alguna tarea de razonamiento o decisión concreta. Cuidado, no reclamamos que sea el deber de Ketelaar y Todd hacerlo; simplemente notamos que dicho apoyo empírico no existe y que el argumento es, así, pobremente soportado.

Nótese la diferencia con la evidencia aportada por otras investigaciones del programa de Heurísticas Rápidas y Frugales, tal como se resumió en el apartado anterior. Allí se brinda evidencia experimental de que el uso de cierta heurística como toma lo mejor genera un alto porcentaje de respuestas correctas en ciertos problemas concretos de elección binaria. Nada parecido ocurre aquí.

\section{IV. ¿Puede Generalizarse la CRÍTICA ANTERIOR?}

Creemos que la crítica particular acerca de la investigación sobre la heurística pico-fin puede ser fácilmente extendida a la mayoría de las investigaciones del programa de Heurísticas y Sesgos. Esto se debe a la metodología utilizada por dicho programa. Considérese la heurística de representatividad presentada anteriormente. Se presenta evidencia de que el uso de dicha heurística provoca varios sesgos como el descuido de la tasa base (base rate neglect). Por ejemplo, retomemos la misma tarea presentada anteriormente (recordemos que se brinda la información de que hay una urna con las descripciones de 30 ingenieros y 70 abogados). Se les presenta a los participantes el siguiente perfil:

Dick tiene 30 años. Está casado pero no tiene hijos. De gran inteligencia, parece que tendrá éxito en su campo. Es muy apreciado por sus colegas.

Probabilidad de que se trate de un ingeniero:

El perfil está creado para que no apunte a ninguna de las dos profesiones. Como resultado de esto, la mayoría de los participantes responden $50 \%$, lo cual es lo esperable si están usando la heurística de representatividad (Tversky y Kahneman 1974, Plous 1993). Sin embargo, tal estimación es reprochable desde el punto de vista normativo. Si la información particular de la descripción 
no apunta a ninguna de las dos posibles profesiones, los participantes deberían utilizar la tasa base dada (30\%) para realizar sus estimaciones.

Así, se presenta evidencia de que, en dicho contexto, los participantes no consideran la información relevante disponible para resolver la tarea. Lo que jamás se ha ofrecido es evidencia de que, en algún otro contexto, el uso de esta heurística tienda a generar respuestas correctas. Es cierto que Tversky y Kahneman y colaboradores usualmente afirman que ese es el caso pero, nuevamente, no se ofrece ninguna evidencia experimental al respecto. Los representantes del programa suelen argumentar que esto se debe a una decisión metodológica, porque en la generación de errores el funcionamiento de la heurística se aprecia mejor. Perfecto, pero eso no altera nuestra crítica: hay una completa ausencia de evidencia empírica de que dicha heurística tienda a generar respuestas correctas en algún contexto concreto.

Nótese que nuestra crítica puede extenderse fácilmente al resto de las investigaciones del programa. Esto se debe, nuevamente, a la metodología empleadapor dichoprograma. Usualmente, cuando sepostula el funcionamiento de una heurística, se propone que toma en consideración cierta información específica, la procesa de tal o cual modo y genera la respuesta. Pues bien, las típicas investigaciones del programa consisten en agregar en la tarea dada a los participantes cierta información normativamente relevante pero que es ignorada por la heurística. Así, la confirmación de que los participantes aplican la heurística postulada genera un error sistemático. Así, globalmente, el programa de Heurísticas y Sesgos, por su forma de trabajar, no suele proveer evidencia positiva que dé cuenta del fenómeno fundamental del problema de marco, es decir, no se provee evidencia de que, para las tareas dadas, en general las personas seleccionan eficientemente la información relevante disponible.

Contrástese el modo de trabajar anterior con el utilizado por el programa de Heurísticas Rápidas y Frugales de Gigerenzer y colaboradores. Allí el foco está, nuevamente, en cómo se acopla el uso de ciertas heurísticas con ciertos ambientes informacionales. Y el punto clave es que usualmente se mide el porcentaje de respuestas correctas que las heurísticas generan, con lo cual se logra aportar evidencia del éxito del uso de las heurísticas estudiadas para resolver tareas concretas. Así, se brinda evidencia de cómo las personas resuelven exitosamente el problema de marco para ciertos contextos concretos.

Resumamos, entonces, el resultado de nuestro análisis. Planteábamos que la psicología cognitiva podía ofrecer aportes sobre cómo las personas de hecho resuelven el problema de marco. Más precisamente, argumentamos que tal disciplina podía ayudar a explicar cómo, ante una tarea dada y mediante la utilización de heurísticas, las personas suelen determinar eficientemente qué información es relevante para realizar la tarea en cuestión. Como el foco del programa de Gigerenzer está, justamente, en el acople de heurísticas a 
determinados ambientes, tal programa suele ofrecer evidencia empírica de cómo la utilización de ciertas heurísticas tiende a lograr resoluciones exitosas de ciertas tareas concretas. Por otra parte, el programa de Tversky y Kahneman se ha enfocado en cómo el uso de heurísticas genera ciertos sesgos o errores sistemáticos. El punto clave es que tales sesgos pueden razonablemente interpretarse como errores al determinar qué información es relevante para resolver la tarea en cuestión. Como consecuencia, este programa suele presentar evidencia empírica de las fallas humanas al determinar relevancia, pero no del éxito típico. Y si bien es cierto que los defensores del programa suelen afirmar que el uso de esas mismas heurísticas provee soluciones adecuadas en otros contextos, no suelen ofrecer evidencia empírica de ello. Así, el aporte de este programa para dar cuenta del fenómeno fundamental del problema de marco es pobremente soportado.

\section{CONCLUSIÓN}

Nuestra breve revisión de la literatura nos deja ver el panorama de la situación. Es cierto que los referentes de los dos programas rivales sobre heurísticas tienen presente, al menos lateralmente, el problema de marco. Sin embargo, es claro que es prácticamente nula la investigación específica sobre el tema. Así, la conclusión más importante de este trabajo es que sería deseable una mayor profundización en la investigación empírica del tema.

Ahora bien, en este contexto de escasez de investigación y ante la pregunta que motiva este trabajo sobre qué programa parece hoy (con la investigación empírica disponible) más adecuado para ofrecer una contribución al problema de marco, nuestro análisis nos permitió concluir que, por la metodología utilizada, el programa de Gigerenzer y colaboradores parecería estar mejor equipado que el de Tversky y Kahneman para realizar dicha contribución.

Es menester, sin embargo, realizar dos importantes clarificaciones antes de finalizar. En primer lugar, la crítica realizada es de hecho y no de principio. Con esto queremos decir que es perfectamente plausible que investigaciones posteriores del programa de Tversky y Kahneman, realizandolas modificaciones metodológicas correspondientes, ofrezcan la evidencia empírica que hoy está ausente. Así, es perfectamente plausible que el programa de Heurísticas y Sesgos pueda en el futuro realizar contribuciones a solucionar el aspecto psicológico del problema de marco. En segundo lugar, las investigaciones típicas del programa de Heurísticas y Sesgos pueden también contribuir a la investigación sobre el problema de marco, aunque sea de manera indirecta. En efecto, como decíamos anteriormente, si bien se acepta que generalmente las personas determinan eficientemente relevancia, se reconoce que en algunas ocasiones se cometen errores. Así, las investigaciones del programa de Tversky y Kahneman podrían contribuir a explicar la ocurrencia sistemática 
de tales errores en la determinación de relevancia. Más radicalmente, las investigaciones de este programa tal vez puedan contribuir a poner en duda la ocurrencia misma del fenómeno fundamental del problema de marco. Esto no ha sido investigado en absoluto y las investigaciones de Tversky y Kahneman son un buen punto de partida para desarrollar preguntas y problemas al respecto.

\section{REFERENCIAS BIBLIOGRÁFICAS}

BLANCHETTE I. \& CAPAROS S. 2013: «When emotions improve reasoning: The possible roles of relevance and utility», Thinking \& Reasoning, 19/3, pp. 399413.

BROWN, F. 1987: The frame problem in artificial intelligence. Proceedings of the 1987 Workshop. Los Altos: Morgan Kaufmann.

CARRUTHERS, P. 2006a: The architecture of the mind: Massive modularity and the flexibility of thought. Oxford: Oxford University Press.

CARRUTHERS, P. 2006b: «Simple heuristics meet massive modularity», en P. Carruthers, S. Laurence \& S. Stich (eds.), The innate mind: Culture and cognition. Oxford: Oxford University Press, pp. 181-198.

CROCKETT, L. 1994: The Turing Test and the Frame Problem: AI's Mistaken Understanding of Intelligence. New Yersey: Ablex Publishing Corporation.

DENNETT, D. 1984: «Cognitive Wheels: The frame problem of AI», en C. Hookway (ed.), Minds, machines \& evolution. Cambridge: Cambridge University Press, pp. $129-152$.

DREYFUS, H. 1979: What computers can't do: The limits of artificial intelligence. New York: Harper Colophon Books.

ELQAYAM S. \& OVER D. 2013: «New paradigm psychology of reasoning: An introduction to the special issue edited by Elqayam, Bonnefon, and Over», Thinking \& Reasoning, 19/3, pp. 249-265.

EVANS, J. 2009: «How many dual-process theories do we need? One, two, or many?» en J. Evans \& K. Frankish (eds.), In two minds In two minds: Dual processes and beyond. New York: Oxford University Press, pp. 33-54.

FODOR, J. 1986: La modularidad de la mente. Madrid: Morata.

FODOR, J. 2008: LOT 2: The language of thought revisited. Oxford: Clarendon Press.

FORD, K. \& HAYES, P. (eds.) 1991: Reasoning agents in a dynamical world: the frame problem. London: JAI Press.

FORD, K. \& PYLYSHYN, Z. 1996: The robot's dilemma revisited: The frame problem in Artificial Intelligence. Norwood: Ablex Publishing Corporation.

GIGERENZER, G. 1996: «On Narrow Norms and Vague Heuristics: A Reply to Kahneman and Tversky», Psychological Review, 103/3, pp. 592-596. 
GIGERENZER, G. 2000: Adaptive Thinking: Rationality in the Real World. Oxford: Oxford University Press.

GIGERENZER, G. 2008: Rationality for Mortals: How People Cope with Uncertainty. New York: Oxford University Press.

GIGERENZER, G. \& BRIGHTON, H. 2009: «Home heuristicus. Why Biased Minds Make Better Inferences», Topics in Cognitive Science 1, pp. 107-143.

GIGERENZER, G., CZERLINSKI, J. \& MARTINGNON, L. 2002: «How Good are Fast and Frugal Heuristics?», en R. Elio, (ed) : Common Sense, Reasoning and Rationality. New York: Oxford University Press, pp. 148-173.

GIGERENZER, G. \& SELTEN, R. 2001: Bounded Rationality: The Adaptive Toolbox. Cambridge. MA: MIT Press.

GILOVICH, T., GRIFFIN, D, \& KAHNEMAN, D. 2002: Heuristics and Biases: The Psychology of Intuitive Judgment. Cambridge: Cambridge University Press.

GLYMOUR, C. 1987: «Android epistemology and the frame problem: Comments on Dennett's cognitive wheels», en Z. Pylyshyn (ed.), The robot's dilemma: The frame problem in artificial intelligence. Norwood: Ablex Publishing Corporation, pp. 65-77.

HJØRLAND, B., 2010: «The Foundation of the Concept of Relevance», Journal of the American Society for Information Science and Technology, 61/22: 217-237.

KAHNEMAN, D. 1999: «Objective Happiness», en D. Kahneman, E. Diener, \& N. Schwarz, (eds). Well-Being: The Foundations of Hedonic Psychology. New York: Cambridge University Press, pp. 3-25.

KAHNEMAN, D. \& FREDERIK, S. 2002: «Representativeness Revisited: Attribute Substitution in Intuitive Judgment», en T. Gilovich, D. Griffin \& D. Kahneman (eds.), Heuristics and Biases: The Psychology of Intuitive Judgment. Cambridge: Cambridge University Press, pp. 49-81.

KAHNEMAN, D., FREDRICKSON, B., SCHREIBER, C., \& REDELMEIER, A. 1993: «When more pain is preferred to less: Adding a better end. Psychological», Science 4, pp. 401-405.

KAHNEMAN, D., \& TVERSKY, A. 1996: «On the reality of Cognitive Illusions», Psychological Review, 103/3, pp. 592-591.

SCHNEIDER, S. 2007: «Yes, it does: a diatribe on Jerry Fodor's The Mind Doesn't Work, That Way», Psyche, 13/1, pp. 1-15.

SHAFIR, E. \& TVERSKY, A. 1992: «Thinking through Uncertainty: Nonconsequential Reasoning and Choice», Cognitive Psychology 24, pp. 449474.

SILENZI, M. I., 2014: El problema de marco: alcances y limitaciones de los enfoques Postcognitivistas. Bahía Blanca: Ediuns.

SILENZI M. I. 2015: «La dualidad del Problema de marco: Sobre interpretaciones y resoluciones», Tópicos: Revista de Filosofía 47, pp. 89-112.

SILENZI, M. I. \& MORO, R. 2015: «Sobre el uso de heurísticas como posible 
KANWISHER, N. 1989: «Cognitive heuristics and American security policy», Journal of Conflict Resolution 33/2, pp. 652-675.

KETELAAR, T. \& TODD P. 2001: «Framing our thoughts: Evolutionary psychology's answer to the computational mind's dilemma», en H. Holcomb (ed.), Conceptual Challenges in Evolutionary Psychology. New York: Kluwer, pp.179-211.

LUDWIG, K. \& SCHNEIDER, S. 2008:«Fodor's Challenge to the Classical computational Theory of Mind», Mind and Language 23/1, pp. 123-143.

MC CARTHY, J. \& HAYES P. 1969: «Some Philosophical Problems from the Standpoint of AI», Machine Intelligence 4, pp. 463-502.

NEWELL, B., WESTON, N. \& SHANKS, D. 2003: «Empirical tests of a fast and frugal heuristic: Not everyone 'takes-the-best'», Organizational Behavior and Human Decision Processes 91, pp. 82-96.

PIATTELLI-PALMARINI, M. 1991: «Probability Blindness. Neither rational nor capricious», Bostonia, Marzo/ Abril:, pp.28-35.

PINKER, S. 2005: «So How Does The Mind Works?», Mind \& Language, 20/1, pp. $1-24$.

PLOUS, S. 1993: The Psychology of Judgment and Decision Making. New York: McGraw-Hill.

PYLYSHYN, Z. (ed.). 1987: The Robot's Dilemma: The frame problem in Artificial Intelligence. Norwood: Ablex Publishing Corporation.

REDELMEIER, D. \& KAHNEMAN, D. 1996 : «Patients’ Memories of Painful Medical Treatments: Real-time and Retrospective Evaluations of Two Minimally Invasive Procedures», Pain 116, pp. 3-8.

SAMUELS, R. 2004 : «Rationality and Psichology», en R. Piers \& A. Mele (eds.) The Oxford Handbook of Rationality. Oxford: Oxford University Press, pp. 279300.

SAMUELS, R. 2005: "The complexity of cognition: Tractability arguments for massive modularity», en P. Carruthers, P. Laurence \& S. Stich (eds.), The innate mind: Structure and contents. Oxford: Oxford University Press, pp. 107-121.

SAMUELS, R. 2010: «Classical computationalism and the many problems of cognitive relevance», Studies in History and Philosophy of Science 3, pp. 280293.

SHANAHAN, M. 1997: Solving the frame problem: A mathematical Investigation of the Common Sense Law of Inertia. Cambridge: The MIT Press.

SHANAHAN, M. 2009: «The Frame Problem». En E. Zalta (ed.), The Stanford Encyclopedia of Philosophy, <http://plato.stanford.edu/archives/win2009/ entries/frame-problem>.

THOMPSON V., EVANS J. \& CAMPBELL J. 2013: «Matching bias on the selection task: It's fast and feels good», Thinking \& Reasoning 19/3, pp. 431-452.

TVERSKY, A. \& KAHNEMAN, D. 1974: «Judgment under Uncertainty: Heuristics and Biases», Science 185/4157, pp.1124-1131. 
Rodrigo Moro es Profesor Adjunto del Departamento de Humanidades de la Universidad Nacional del Sur (Argentina) e Investigador del Instituto de Investigaciones Económicas y Sociales del Sur (IIESS-CONICET).

\section{Lineas de Investigación:}

Filosofía de la Ciencia; Cuestiones metodológicas en psicología cognitiva y economía conductual

\section{Publicaciones recientes:}

MORO, R., FREIDIN, E., y TOHMÉ, F. (2015): «Social preferences are not enough: Accounting for anomalous behavior in a complex mixed-motive game», Cuadernos de Economía 34(65), pp. 261-278.

HASRUN, H., FREIDIN, E. y MORO, R. (2015): «Las normas sociales en los experimentos de coima: la necesidad de explicitar el estatus normativo y los deberes de cada rol», Revista Argentina de Ciencias del Comportamiento 7(2), pp. 41-58.

Correo electrónico: rmoro@uns.edu.ar

María InÉs Silenzi es Asistente de Docencia del Departamento de Humanidades de la Universidad Nacional del Sur (Argentina) e Investigadora del Instituto de Investigaciones Económicas y Sociales del Sur (IIESS-CONICET).

Lineas de Investigación:

Problema de marco; Filosofía de las Ciencias Cognitivas

\section{Publicaciones recientes:}

SILENZI, M. I. y MORO, R. (2015): «Sobre el uso de heurísticas como posible solución del problema de marco», Crítica 47 (140), pp. 65-91.

(2015): «La dualidad del problema de marco: Sobre interpretaciones y resoluciones», Tópicos 47, pp, 89-112.

Correo electrónico: misilenzi@uns.edu.ar 